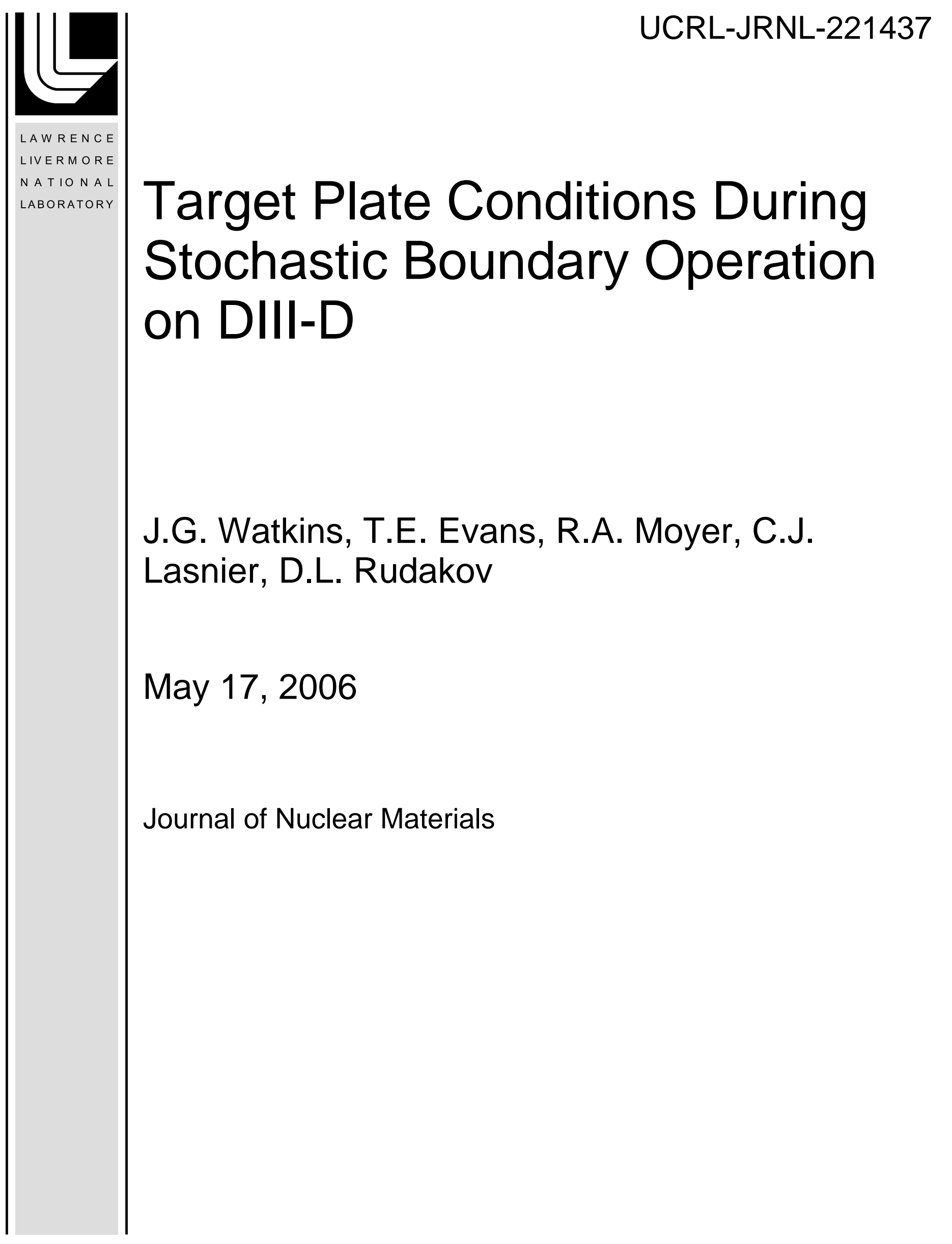


This document was prepared as an account of work sponsored by an agency of the United States Government. Neither the United States Government nor the University of California nor any of their employees, makes any warranty, express or implied, or assumes any legal liability or responsibility for the accuracy, completeness, or usefulness of any information, apparatus, product, or process disclosed, or represents that its use would not infringe privately owned rights. Reference herein to any specific commercial product, process, or service by trade name, trademark, manufacturer, or otherwise, does not necessarily constitute or imply its endorsement, recommendation, or favoring by the United States Government or the University of California. The views and opinions of authors expressed herein do not necessarily state or reflect those of the United States Government or the University of California, and shall not be used for advertising or product endorsement purposes. 


\title{
Target Plate Conditions During Stochastic Boundary Operation on DIII-D*,
}

\author{
J.G. Watkins ${ }^{\text {a }}$, T.E. Evans ${ }^{\text {b }}$, R.A. Moyerc, C.J. Lasnierd, and D.L. Rudakov ${ }^{c}$ \\ ${ }^{a}$ Sandia National Laboratories, Albuquerque, New Mexico 87185-1129, USA. \\ ${ }^{b}$ General Atomics, San Diego, California 92186-5608, USA \\ ${ }^{c}$ University of California, San Diego, La Jolla, California 92093-0417, USA \\ ${ }^{d}$ Lawrence Livermore National Laborabory, Livermore, California 94550, USA
}

\begin{abstract}
A major concern for large tokamaks like ITER is the presence of edge localized modes (ELMs) that repeatedly send large bursts of particles and heat into the divertor plates. Operation with resonant magnetic perturbations (RMP) at the boundary of DIII=D has suppressed ELMs for values of q95 3.7. At the target plate, the conditions during ELM suppressed operation for both high and low collisionality are observed by a set of radially distributed Langmuir probes. At high collisionality $(\nu * \sim 1)$, the target plate particle flux and temperature drops by $>30 \%$ during ELM suppression. At low collisionality $\left(v^{*} \sim 0.1\right)$, the core density, target plate density, and target plate particle flux drop but the plate electron temperature increases after the ELMs are suppressed. The ELM-suppressed target plate heat flux is nearly the same as the heat flux between ELMs but the (5X higher) transient heat flux peaks due to ELMs are eliminated.
\end{abstract}

JNM keywords: List up to 5 Journal of Nuclear Materials keywords

PSI-16 keywords: List up to 5 PSI keywords

PACS: List up to 4 Physics and Astronomy Classification Scheme (PACS) codes

\footnotetext{
*Work supported by the U.S. Department of Energy under Contract Nos. DE-AC04-94AL85000, De-AC0399ER54463, W-7405-ENG-48, and Grant No. DE-FG03-95ER54294.
} 
*Corresponding author address: J.G. Watkins, c/o General Atomics 13-350, P.O. Box 85608,

San Diego CA 92186

*Corresponding author e-mail:watkins@fusion.gat.com

Presenting author address: same as above

Presenting author e-mail: same as above 


\section{A. Introduction:}

Unacceptable erosion and thermal loading of the divertor plates are predicted [1,2] for Type 1 ELMing H-mode operation in large tokamaks like ITER. One possible solution, that has been recently demonstrated $[3,4,5,6]$ in DIII-D, is operation with resonant magnetic perturbations (RMP) at the boundary. These plasmas exhibit ELM suppression under certain conditions while maintaining high energy confinement. This magnetically perturbed boundary is produced by a special set of coils (I-coils) that generate small resonant perturbations of the edge magnetic field. The RMPs and ELM suppression effects exhibit a resonance behavior centered around a particular edge $q$ value $\left(q_{95}=3.7\right)$. At the target plate, the conditions during ELM suppressed operation are observed by a radial array of Langmuir probes for both high and low collisionality cases.

\section{B. Experimental Setup:}

Double null plasma discharges with high triangularity (high density, high collisionality) and low triangularity (low density, low collisionality) were magnetically biased down toward the lower divertor for these experiments. The low collisionality case could only be obtained at low triangularity because the outer strike point needed to be located near the baffle entrance of the outer divertor cryopump to bring the core density down to the desired level. In these experiments, the grad B drift was directed toward the main (lower) divertor. Both cases have similar edge q but have different densities. The edge q was varied by changing the plasma current with constant toroidal field.

The resonant magnetic perturbation at the boundary was generated using an installed set of rectangular coils internal to the DIII-D vacuum vessel. These coils are mounted under the outer wall graphite tiles and were energized with up to $4.5 \mathrm{kA}$ of current during plasma discharges. The polarity of the direct current in these coils could be varied to control the up/down symmetry or spatial location of the perturbation fields. There are two sets of 6 coils 
distributed around the inside of the vacuum vessel with one set in the upper and one set in the lower outer walls of DIII-D. In these experiments, we used the coils to drive $n=3$ perturbations with up/down symmetric (even parity) or asymmetric (odd parity) perturbations. For high collisionality, the odd parity was more effective for ELM suppression and for low collisionality, the even parity the more effective suppressing the ELMs (Figure 1).

Target plate measurements were made during and after the transition to the ELM suppressed regime using a fixed radial array of Langmuir probes. The probe voltage sweeping was at $1250 \mathrm{~Hz}$ to improve time resolution and accuracy during ELMs and oscillations. Details of the DIII-D Langmuir probe system have been described previously $[7,8]$.

\section{Observations:}

\section{High collisionality}

At high collisionality ( $(* \sim 1)$ and q95 fixed near optimum, comparing characteristic values when the perturbation coil is energized to the value between ELMs, the target plate particle flux drops by $30 \%$, the temperature drops by $50 \%$, and the probe floating potential $\left(\mathrm{V}_{\mathrm{f}}\right)$ goes to approximately zero across the floor. These changes indicate that the plasma is tending toward detachment. Also at high collisionality, small oscillations at about the ELM frequency but with amplitude clamped at a value much smaller than the ELM level are observed in temperature, density, and $\mathrm{H}_{\alpha}$ at the target plate after the ELMs are suppressed (figure 2). The ELM peak heat flux is reduced by a factor of at least 5 at the divertor plates as measured by the infrared camera [4].

The peak particle flux measured during an ELM before the transition drops by a factor of $\sim 10$ after the RMP is energized and the ELMs are suppressed. The transition is shown in figure 2. The figure shows that, $2 \mathrm{~cm}$ outboard of the outer strike point, the temperature and the particle flux drop at the transition. For these cases with near optimum ELM suppression, the target plate electron temperature (baseline/between ELMs) is observed to drop by $30-50 \%$ 
within $20 \mathrm{~ms}$ after the perturbation coils are energized. The target plate particle flux (between ELMs) decreases by 30 percent at this transition.

The floating potentials measured at three divertor plate locations changed when the coils are energized. Three $\mathrm{V}_{\mathrm{f}}$ measurements were at 2,5 , and $8 \mathrm{~cm}$ outside the outer strikepoint. Two of these (5 and 8) go to zero at the transition where the RMP coils are energized. This is an indication that the temperature has decreased in the outer SOL and that there is little sheath acceleration of ions into the plate in this region. This is consistent with lower levels of heat flux at the plates.

Resonant behavior in $q_{95}$ is observed in the target plate conditions as well as in the effectiveness of the RMP to suppress ELMs. The time history of the q scan can be seen in figure 3. The ELM suppression q window is shown in the particle flux contour plot measured at the lower divertor plate. The $\mathrm{H}$ alpha signal measured in the same region (FS03) is shown for reference. During a scan of $q_{95}$, obtained by ramping up the plasma current during resonant magnetic perturbation operation, the between ELM target plate particle flux was maximized and the target plate electron temperature is minimized at $q_{95}=3.7$, the same resonant value required for optimum elm suppression. The edge q dependence of the particle flux, electron temperature, and density can be seen in figure 4 . Since the particle flux is maximized at the optimum $\mathrm{q}_{95}$, the $30 \%$ drop in particle flux at the transition is really a minimum and the drop is larger at other $\mathrm{q}$ values. The window for suppressing ELMs can be seen centered around $\mathrm{q}_{95}$ $=3.7$.

Unique oscillations are observed at the target plate during ELM suppressed RMP operation. The oscillations first appear about $50 \mathrm{msec}$ after the start of the I-coil pulse at 3000 msec into the discharge and can be seen on the divertor $\mathrm{H}$ alpha as well as the target plate flux and temperature as shown in figure 2. The oscillations in particle flux and temperature measured by the Langmuir probes increase and then decrease together in time. 


\section{Low collisionality}

In low collisionality plasmas ( $v_{* \sim 0.1}$, after the ELMs are suppressed, the core density drops, the target plate particle flux drops by $25 \%$ while the electron temperature at the plate increases by about $100 \%$. We observe the floating potential at the strike point (Rprobe $=$ Rstrike $+0.1 \mathrm{~cm}$ ) drop strongly after the ELMs are suppressed to $-150 \mathrm{~V}$. At low collisionality, we also observe a visible light emission pattern across the divertor floor that may correspond with tile edges. The perpendicular heat flux during the ELM suppressed phase is about $15 \mathrm{~W} / \mathrm{cm} 2$ between ELMs before the transition and $20 \mathrm{~W} / \mathrm{cm} 2$ after the transition. The Langmuir probe heat flux agrees with the IR camera if a sheath factor of 1 is used as shown in figure 5. So the background heat flux does not change much after the I-coil is energized but the transient heat flux peaks due to ELMs (5X higher than between ELMs) are eliminated. No oscillations of the type seen in high collisionality are observed.

\section{Discussion:}

\section{High Collisionality}

During RMP operation at high collisionality, the peak values of the particle flux and temperature at the target plate drop significantly because of the disappearance of most of the ELMs. Additionally, the baseline of particle flux and temperature decrease after the start of the I-coil pulse and the floating potential drops to zero. These baseline effects are more what we expected to see with the resonant magnetic perturbation coils. We expected to lower the divertor temperature and density by spreading out the normal profiles over a larger area due to the modifications in the flux surface geometry. The 30\% drop in particle flux and 50\% drop in temperature implies that the baseline heat flux after the transition should be about 4 times lower than the heat flux measured between ELMs before the transition. The temperature reaches a minimum when the edge $\mathrm{q}$ is adjusted to maximize the particle flux. This should also minimize the steady state heat flux. These trends are leading toward divertor detachment if not already achieved. More detailed profiles with data closer to the strike point are needed 
to determine if this is the case. Even at $2 \mathrm{~cm}$ into the $\mathrm{SOL}$, we see the temperature drop to less than $5 \mathrm{eV}$. The oscillations which occur at about the same rate as the former ELMs but with reduced or clamped ampitude may be evidence of particle and energy bursts which are spread out in time due to the more complicated field geometry. The oscillations have a longer duration and slower rise time than the ELMS but the integral of the particle flux is about the same as during the ELMS thus the particle and heat impulse is substantially reduced due to the longer period of the oscillations. It is the high impulse that is predicted for ITER like ELMS that erodes or ablates particles.

\section{Low Collisionality}

At low collisionality, more typical of ITER conditions, the observation of higher baseline electron temperature at the target plate is typical of lower density operation where radiation losses are smaller in the divertor because of reduced recycling. The very high floating potential measured also indicates the presence of higher temperature or hot electrons which carry heat directly and more efficiently to the target plate from upstream or possibly even from deep inside the steep pedestal plasma gradient region. Field line tracing calculations [9] predict that a few percent of the core field lines do actually wander out of the core and into the target plate for larger magnetic perturbations such as were used in the low collisionality ELM suppression cases. These hot electrons could produce higher localized heat flux on tile edges where field line incident angles are higher. This type of heat flux issue can be dealt with by tapering the edge surfaces to reduce the incident angles or by reducing the tile gaps and improving alignment as we have done with the new DIII-D lower divertor tiles which will be used during the 2006 operating campaign. Another possible solution would be to raise the divertor density to increase radiation losses. We will try to run higher density and saturate the wall to simulate ITER divertor conditions in future experiments.

\section{E. Conclusions:}

RMP operation, by suppressing ELMs and significantly reducing heat and particle fluxes 
at the target plates, greatly improves the outlook for operating large $\mathrm{H}$ mode fusion reactors. The high collisionality experiments described here show that the baseline levels of particle flux and electron temperature drop after the transition and depend on the edge $q$ as does the resonant magnetic perturbation. The maximum particle flux and minimum temperature occur at the optimum q for ELM suppression and imply that this $q$ is where the perturbations have the most effect. By applying the RMPs at low collisionality, the impulsive heat loading during ELMs has been converted to a more steady-state heat loading which keeps the peak heat flux below the expected ITER ablation limit (5-10\% of Wped). The higher target plate heat flux we see at low density from hot electrons should have less of an effect on tile edges with reduced tile gaps and improved tile alignment.

Further work needs to be done to understand the physics mechanisms and control parameters leading to the ELM suppression, to explore the operational space available, and perhaps find a more optimized coil configuration.

\section{F. References:}

[1] A. W. Leonard, J. Nucl. Mater. 266-269 (1999) 109

[2] A. W. Leonard, APS invited talk - phys of plasmas

[3] T.E. Evans, R.Moyer, P. Thomas, J.G. Watkins, et al, Phys. Rev. Lett. 92 (2004) 235003-1

[4] T. E. Evans, R. A. Moyer, J. G. Watkins, et al, Nuc. Fusion $4 \underline{5}$ (2005) 595

[5] T. Evans, J. Nucl. Mater. 1-2 (1993) 160.

[6] K. Burrell, et al, PPCF 47 (2005) B37

[7] J.Watkins, et.al., J. Nucl. Mater. 241-243 (1997) 645

[8] D. Buchenauer, et.al. RSI. 61 (1990) 2873

[9] L. Yan, 2006 PSI (this conference) 


\section{E. Figure captions:}

Figure 1

The high (dashed) and low (solid) collisionality ELM suppression cases are shown. The low collisionality case is similar to ITER pedestal collisionality and is achieved with a larger magnetic perturbation using even parity. This case also had more effective Elm suppression, lower pedestal density and higher target plate temperatures.

Figure 2

High Collisionality target plate conditions during ELM suppression using the resonant magnetic perturbation (RMP). Particle flux and temperature at Rsep $+2 \mathrm{~cm}$ are compared before and after the transition. The baseline level of particle flux drops $30 \%$ and the baseline level of electron temperature drops $50 \%$ when the coil is energized. Small oscillations are seen in target plate density and temperature as well as $\mathrm{H}$ alpha light after the transition.

Figure 3

The figure shows a contour plot of the target plate particle flux during the q scan. The vertical axis is major radius and the horizontal axis is time for all the traces. The RMP is energized at $3000 \mathrm{msec}$ (bottom trace vs time) and the ELM suppression is seen where the edge $\mathrm{q}$ is within the suppression window (around 3.7). Several events can be seen later in the discharge which may be a result of q decreasing out of the resonant window.

Figure 4

Conditions at the target plate $2 \mathrm{~cm}$ outboard of the outer strikepoint are shown as a function of edge q. The ELM suppression can be seen in a window around $\mathrm{q} 95=3.7$. The maximum target plate particle flux and minimum target plate electron temperature occur at a particular edge q near the center of the ELM suppressed window.

Figure 5 
Low collisionality target plate conditions are shown for a Langmuir probe near the strike point (Rstrike $+0.1 \mathrm{~cm}$ ). The electron temperature goes up and the target plate flux goes down when the coil is energized at $2000 \mathrm{msec}$. The Elms go away at $2350 \mathrm{msec}$.

Figure 6

At low collisionality, the floating potential goes very negative when the ELMs go away. This is consistent with hot electrons hitting the target plate. The floating potential at the inner strike point is slightly positive. 


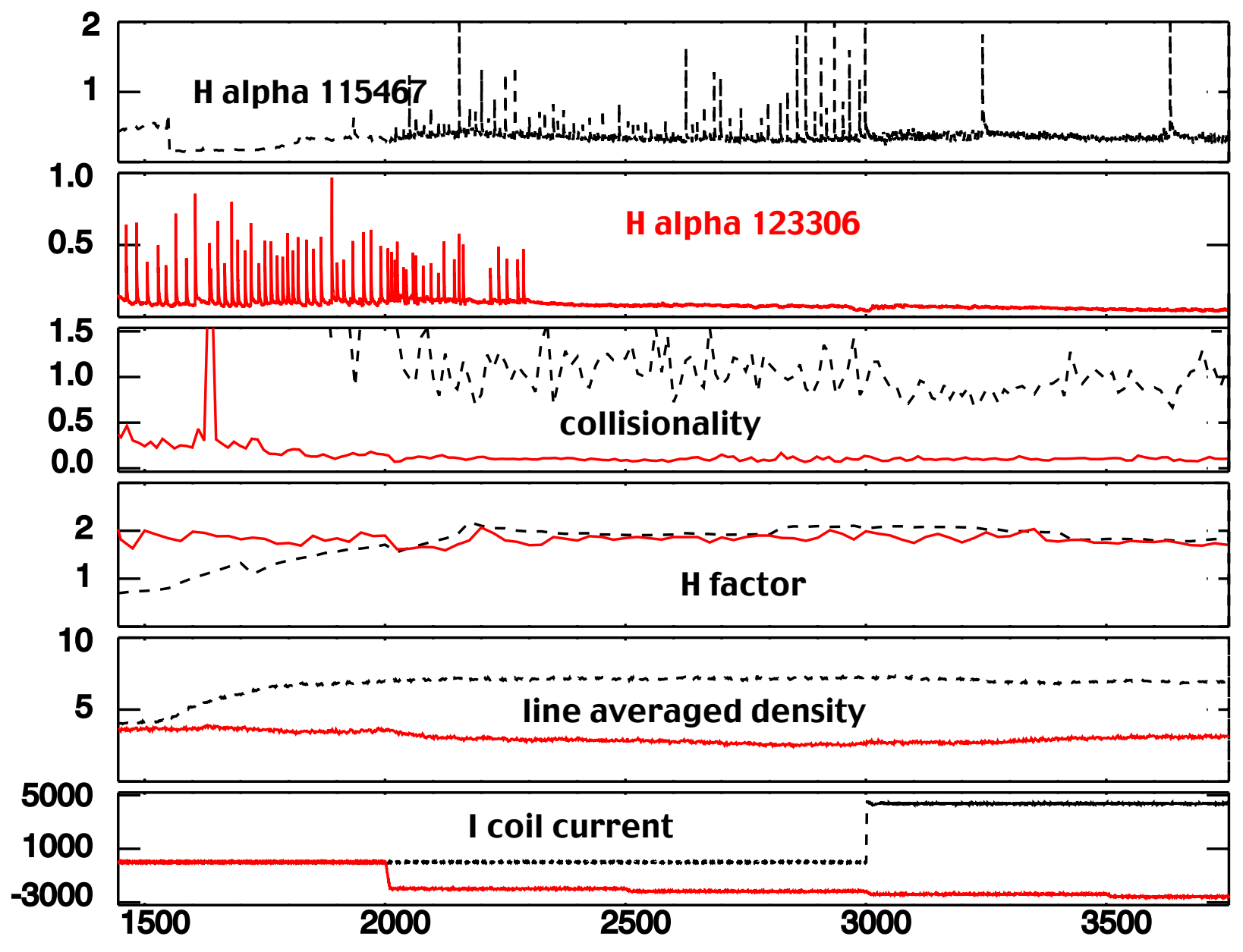

Figure 1 


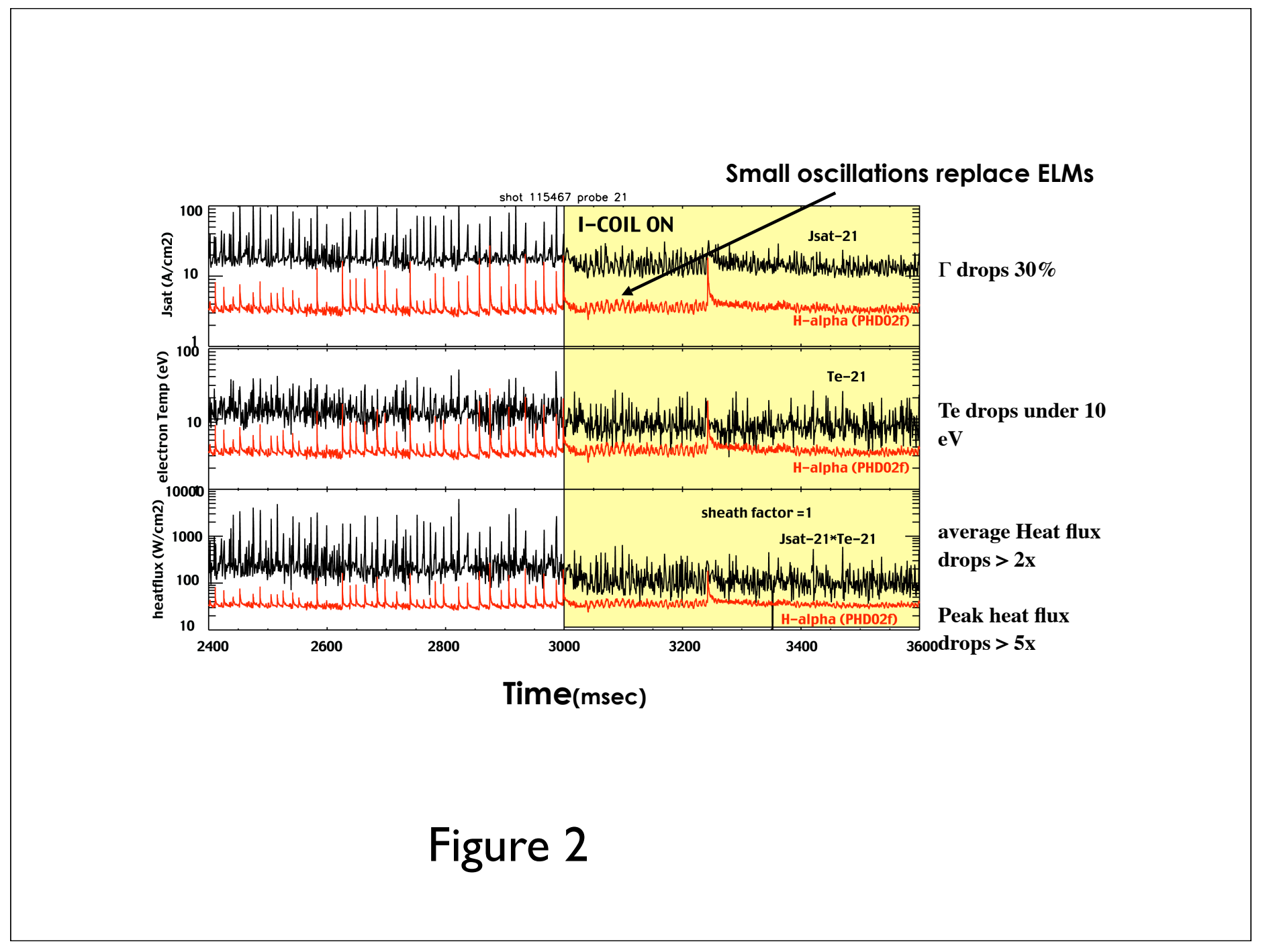




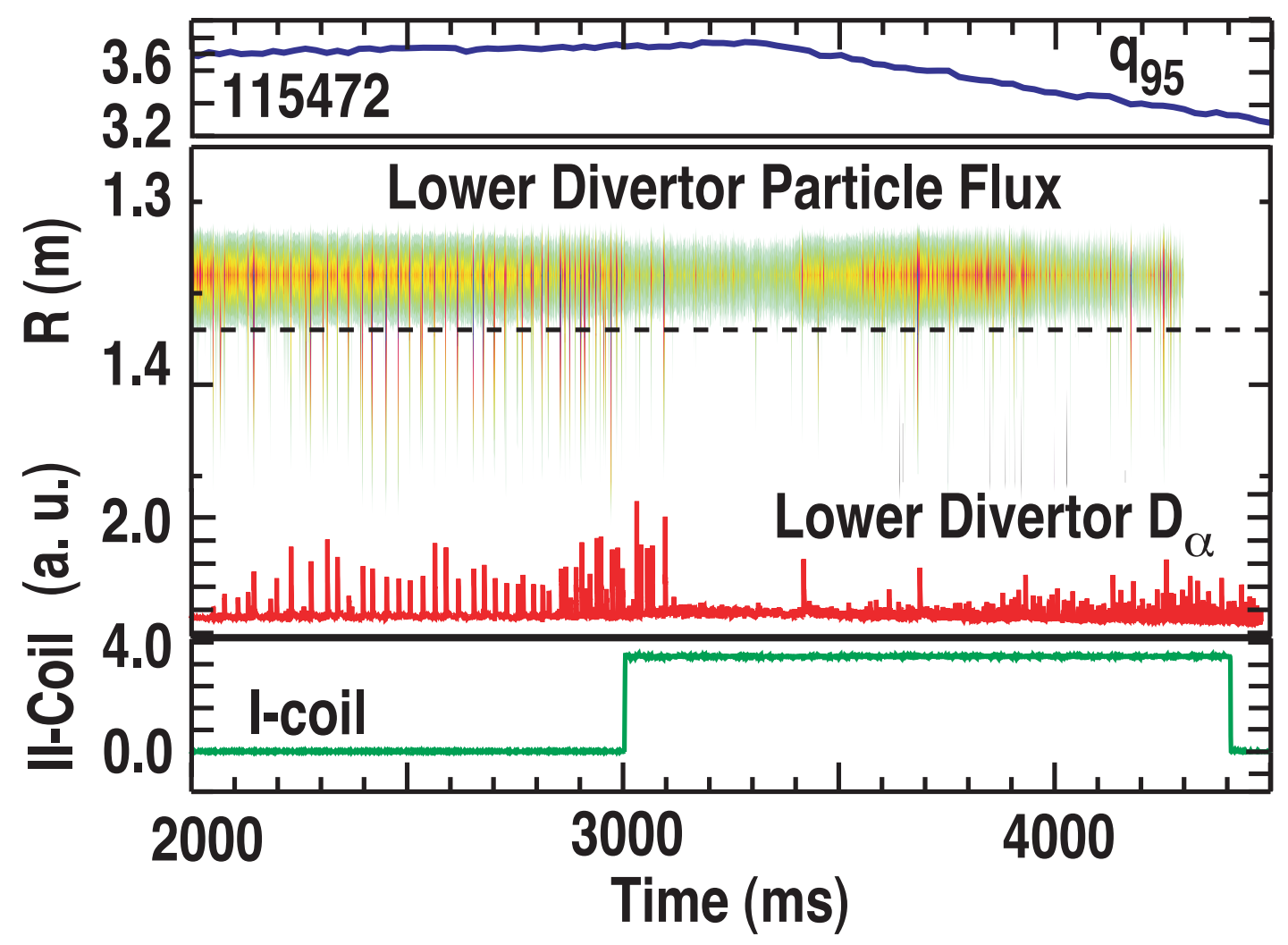

Figure 3 

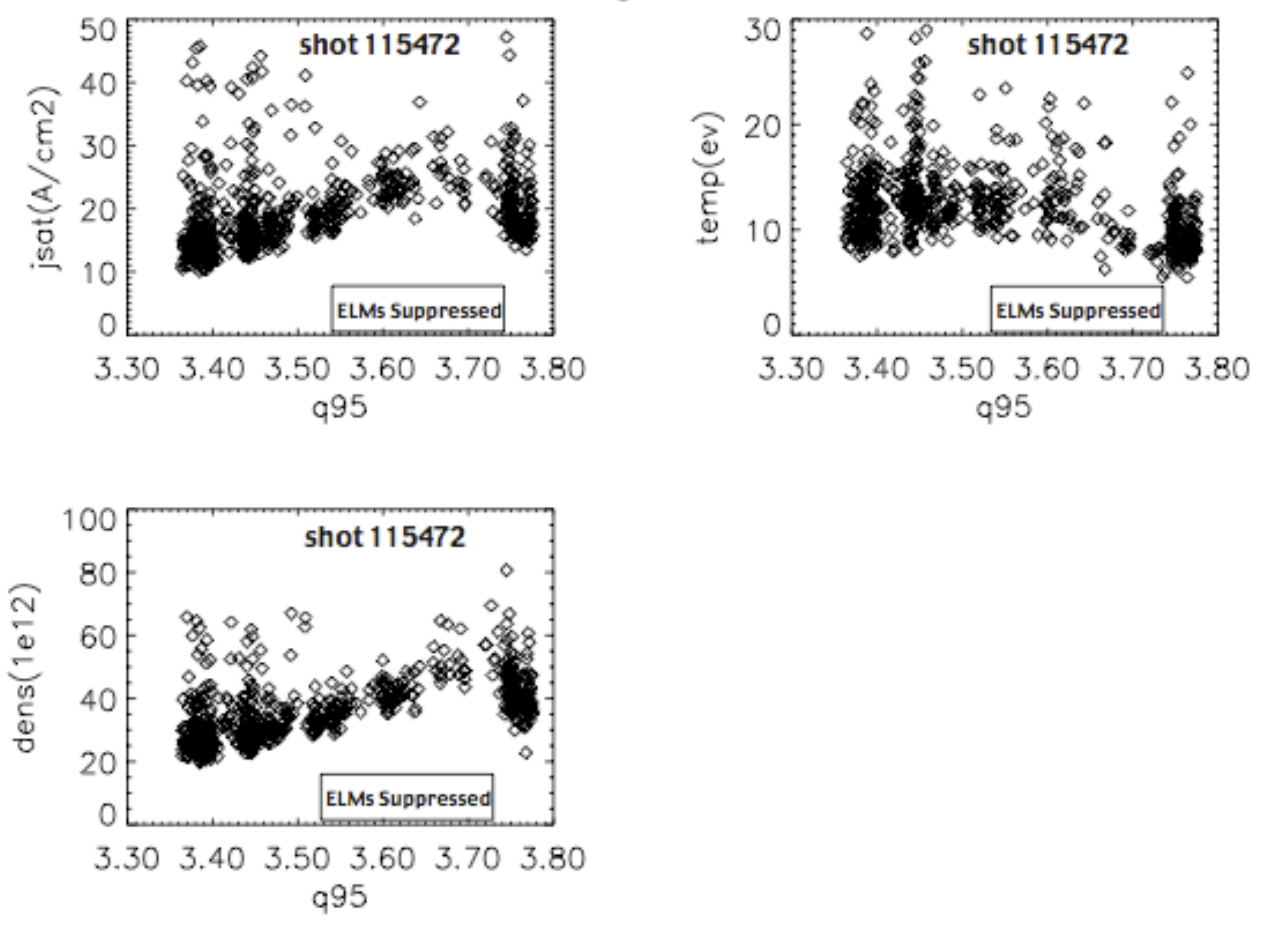

Figure 4 


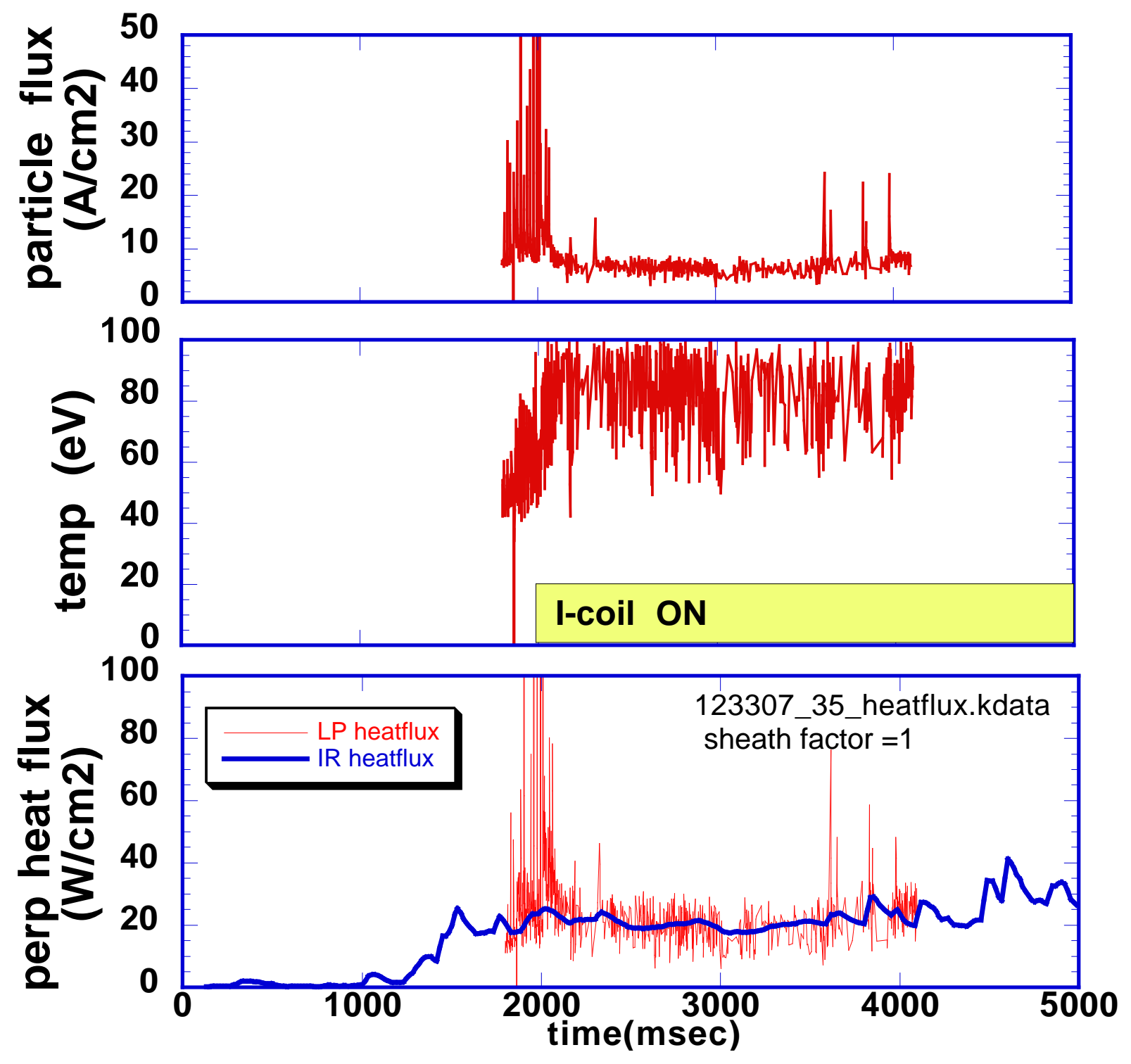




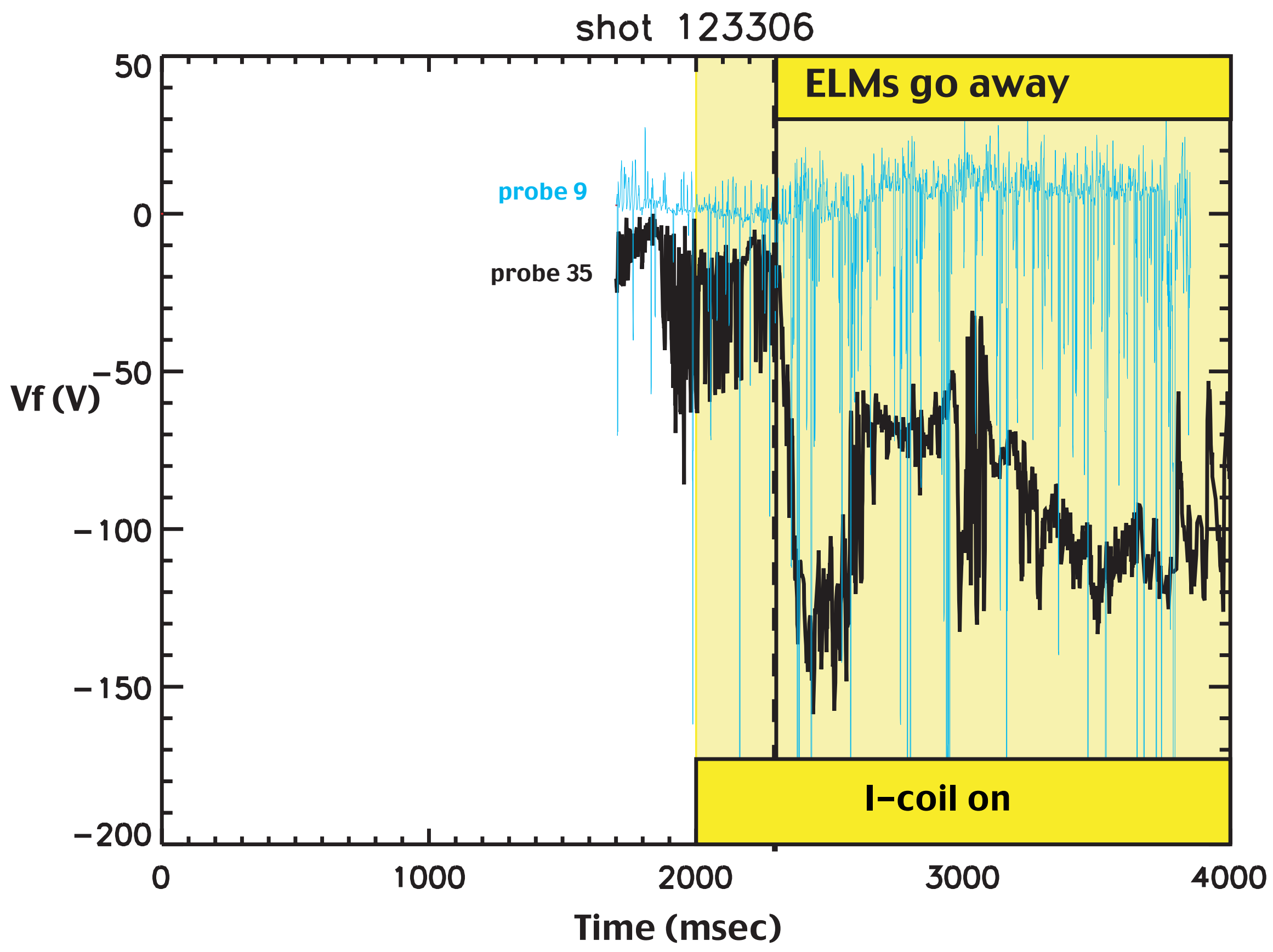

